\title{
Study of the mobile number portability (MNP) implementations in Belarus and Georgia
}

\author{
[ Armen Ghalumyan ]
}

\begin{abstract}
Mobile number portability (MNP) is considered an important condition for ensuring full competition in the electronic communications sector, allowing mobile subscribers to change their service providers any time without altering their phone numbers. Mandated in the European Union since 2003, experience of MNP implementations across the EU member states has been largely successful. On the contrary, introduction of MNP in the post-Soviet region has been rather slow, where Belarus and Georgia pioneered with implementation. After the collection of primary data among relevant stakeholder groups and the subsequent analysis thereof, the given paper provides key revealed findings and lessons learnt from MNP implementations in those two countries, as well as an overview of any impact the MNP service availability has had on the Georgian and Belarusian electronic communications markets.
\end{abstract}

Keywords-mobile number portability, Belarus, Georgia, telecommunications, mobile service, market competition

\section{Introduction}

Mobile number portability (MNP) has been mandated for all states across the European Union since 2003, and their experience is largely considered a success story in increasing consumer choice and market competition as well as providing other benefits to sector stakeholders. On the contrary, developing countries, including those from the former Soviet Union, are making their first steps in introducing number portability. The given paper presents main findings of the study of MNP effects on electronic communications markets of the two post-Soviet countries, Belarus and Georgia. These were the first states in the post-Soviet region to launch MNP in 2011 and 2012, respectively. In fact, launch of the number portability service in Georgian mobile communication networks entered history as the smoothest and quickest solution ever. It was deployed within 4 months; as of now, it is one of the most quickly implemented solutions in the whole history of implementations. Some other peer countries from the post-Soviet space, such as Moldova, Azerbaijan, Russia, Armenia and Kazakhstan, followed the trend with certain delays, whereas the remaining ones still debate around the issue.

As with any other service, the demand for MNP is directly correlated with its high usage rates. Policy makers around the world heavily rely on porting statistics as a measure of success of the MNP implementations. The annual data on ported numbers are defined as an indicator by International Telecommunication Union (ITU) and are regularly collected

Armen Ghalumyan

Telecom Ecole de Management

France for the latter's World Telecommunication/ ICT Indicators database. It is regarded as a noteworthy benchmark also by the European Commission, which includes number portability statistics in its Digital Progress Report, as a dedicated section of the country profiles for each of the EU member states. In his work on the MNP lessons from international experience, Rohan Samarajiva (2013) acknowledges that "in the short term low porting rates may mean failure" and further suggests a formula for the MNP success: "High porting rates = costrecovery $=$ increase churn $/$ improve competition $=\mathrm{MNP}$ success." At the same time, however, as experience of certain countries shows, high demand for MNP might not necessarily equate to the attained improvements like increased customer satisfaction. Furthermore, although most of the available literature associate the success of MNP with high porting rates, some papers argue that the service implementation could still be successful under low rates of number portings. For instance, Tahani Iqbal (2010) claims that this is likely to be the case if MNP leads to improved market competition resulting in lower tariffs, better services and hence satisfied customers; therefore, she concludes that "the argument for high porting rates being the sole indicator for the success of the MNP service is erroneous." The same is confirmed by Larkotey et al. (2012) in their case study of MNP successes and failures in a number of developing countries from the West African subregion.

When it comes to porting statistics for the two countries in question, Georgia had reached more than 107 thousand number portings in just one year since the service launch, whereas in Belarus the number of mobile subscribers that ported their numbers in the first 4 months after the introduction of MNP amounted to some 2,000 people, constituting only $0.019 \%$ of the country's total subscriber base of 10.7 million. The question arises here: do these figures indicate that the MNP implementation was successful in Georgia but rather unsuccessful in Belarus? Absolutely not, as the number of portings alone is an insufficiently adequate criterion for the impact of the MNP implementation. It is merely a simple reference for policy makers to quantify the demand for MNP, which should be accounted for throughout the entire period since the service introduction and not at a single point in time, together with some other quantitative as well as qualitative aspects. It implies a holistic insight into the effects of MNP on different stakeholder groups, such as final consumers, regulatory or policy-making bodies, and mobile operators.

The given research paper puts forward a multi-stakeholder data gathering approach and subsequent analysis of the revealed findings, which can provide useful inferences to assess the impact of MNP on the electronic communications markets of Georgia and Belarus. Through the prism of primary and secondary data analysis, it looks at views and perspectives 
of the key parties concerned, which, when taken cumulatively, attempts to ensure a comprehensive picture on whether MNP has resulted in any positive effect.

\section{Description of the data collection instruments}

The given research presented herein has envisaged studying views and perceptions regarding the MNP implementation of the following three stakeholder categories in Belarus and Georgia:

- National regulatory authorities (NRAs)/ policymaking bodies in charge of the telecommunications field;

- $\quad$ Acting mobile operators;

- Ordinary mobile phone users, irrespective of whether they have ever had MNP experience or not.

For that purpose, separate data collection tools (or questionnaires) were designed for each of the above categories. In addition to a specific questionnaire for mobile operators, a data request sheet was prepared for this stakeholder group to ask for certain numerical data and subsequently analyze if MNP has had any impact on a number of variables, such as the companies' market shares, revenues from mobile services, average per-minute charges for on-net and off-net calls, etc.

All questionnaires and the data request sheet for mobile operators were drafted in English and then translated into Russian, as this language is quite widely used in both of the studied countries, especially in Belarus. Structured interviews (either face-to-face or via Skype) were chosen as a data gathering method for NRAs/ policy-making bodies and mobile operators. In contrast, for the mobile users' audience where a considerably higher number of respondents was required, an online survey approach was decided upon. For the latter, the online form builder JotForm was used.

The questionnaires contained a combination of open- and closed-ended questions, aimed at revealing respondents' feedback on a broad range of aspects related to their MNP exposure. The ones for NRAs/ policy-making bodies and mobile operators consisted mostly of open-ended questions, requesting them to freely express their thoughts at an interview, each to last for approximately an hour. Besides, some questions addressed to these two stakeholder groups were common, as they were expected to closely cooperate on the MNP implementation project but to have potentially opposing views around the same issue.

On the contrary, there were mainly closed-ended questions in the mobile user survey, to take a maximum of 10 minutes to complete. During consumer surveys of this sort, respondents usually don't bother writing lengthy answers; therefore, predominantly multiple-choice questions, often providing numerical ranges for possible answers, were included. The online questionnaire was also constructed in a way to account for the so-called conditional logic, whereby depending on the respondents' answers to a preceding question, one or several subsequent questions would be skipped as non-applicable, thus shortening the questionnaire fill-in time. For instance, if the survey respondent has never used the MNP service, several questions related specifically to the MNP usage experience would not appear at all.

The next section provides an overview of the data collection process in both countries.

\section{Description of the data collection and analysis process}

The data gathering process among institutional stakeholders, i.e., NRAs/ policy-making bodies and mobile operators, took place during the below mentioned periods:

- Georgia: June 9-11, 2015

- $\quad$ Belarus: June 24 - July 8, 2015

For Georgia, all 5 interviews ( 3 with mobile operators and 2 with the NRA and a policy-making body) were conducted in a face-to-face mode at the interviewees' premises in Tbilisi. For Belarus, 2 interviews with one of the mobile operators and the Ministry of Communications and Informatization (MCI) were held via Skype, whereas another mobile operator responded to the questionnaire in writing and provided its feedback by email. The remaining third mobile operator didn't respond to several email requests to participate in the survey, in spite of the fact that the MCI asked them to do so and the company replied positively to the Ministry's mediation. All interviews were recorded upon the consent of interviewees, and based on those recordings the summary of stakeholder interviews was prepared.

None of the respondent mobile operators agreed to fill in the data request sheet, refusing to do so on the grounds that the requested information was of a confidential nature. The Georgian mobile operators claimed that they periodically submit some of the required data to the NRA, which are available on the latter's website. For Belarus, no such a unified public source was available at all and so the analysis was further complicated. Therefore, the limited corporate-level data, compared to what was asked for in the data request sheet, made it difficult to analyze the impact of MNP on the performance of individual companies in particular and of the larger mobile telephony market in general.

As for the consumer survey, the initial questionnaire prepared by means of the online form builder JotForm had been tested during a couple of weeks in October 2015. As a result, about 30 completed questionnaires were collected from both countries after disseminating the survey among personal contacts and via social media platforms, such as Facebook. Those responses were then analyzed and the questionnaire was correspondingly modified to take account of findings and lessons learnt from the piloting stage. Based on the revised questionnaire, the main consumer survey phase commenced on February 29, 2016 and lasted for 7 months until September 26, 2016. Again, the online survey was promoted through the network of personal contacts with the heavy use of social 
media. During that period, 255 completed questionnaires were gathered from Belarus and 190 from Georgia, comprising respectively $0.003 \%$ and $0.005 \%$ of the countries' populations $(9.5 \mathrm{~m}$ and $3.9 \mathrm{~m})$. The total of 445 survey responses were then exported into an Excel spreadsheet, which was used as a dataset for the analysis of mobile users' MNP perceptions and experiences.

As a next step, the dataset was coded and run through the SPSS application to generate cross-tabulations across different survey questions as well as with the use of all demographic dimensions included in the questionnaire, such as age, gender, marital status, level of education, current occupation, and monthly personal income. The resulting output tables showed how responses to the survey questions are distributed per each of the above dimensions, e.g., to reveal if the MNP usage is any sensitive to age or level of income. Moreover, response rates to cross-tabulated questions might exhibit some signs as to whether an explainable pattern is detected, e.g., if a high percentage of mobile customers that are happy with current service providers are indeed less willing to port their phone numbers. Ultimately, the produced cross-tabulations were analyzed with an attempt to arrive at certain conclusions regarding the survey respondents' mobile usage and MNP experiences, separately for Belarus and Georgia.

\section{Discussion and conclusions}

In accordance with international experience, the introduction of MNP is aimed at benefitting final consumers and mobile communications markets, as it is expected to bring about various socioeconomic effects relating to the increased wellbeing of mobile users as a result of enhanced consumer choice and inter-operator mobility. For commercial players, the MNP availability is an additional means to gain new customers and a good stimulus to shake up their strategic and operational models to adapt to a changing competitive environment. So far, the worldwide MNP track record has seen varying implementation practices and outcomes, ranging from largely successful to nearly unnoted and depending on a great deal of particular country and market specifics.

This section discusses key findings of the multistakeholder analysis of MNP implementations in Belarus and Georgia, in an effort to derive inferences as to whether those have had any profound impact on the national electronic communications markets. Where applicable, references are made to findings of the academic literature review on previous MNP research, which formed part of the given study.

In both countries, the MNP project was initiated by the national telecoms regulator or policy maker, pursuing a range of specific objectives. In addition to the government authorities, the latest entrant mobile operators were the only supporters of the idea. The governmental stakeholders are of the opinion that their expectations from the MNP introduction have largely or partially been achieved, leaving an overall positive impact on the sector's development and strengthening of competition. As to mobile operators, their views on the effects of MNP are mostly neutral or skeptical, even those of the latest entrants that were actively lobbying for the service during its pre-launch period and were expected to reap much needed benefits from it. They believe that MNP has been mostly insignificant for the performance of their companies in particular and of the mobile services market in general.

The MNP facilities in both Belarus and Georgia are in line with nearly all parameters for an ideal MNP framework, identified by the international best practice research. In Belarus, the related technical approach and specific attributes have changed over time, from a donor-led, fee-based lengthy process in the early years of MNP to a recipient-driven freeof-charge porting regime of reasonable duration that is available nowadays. So, from the technical standpoint these two deployments can be considered effective and compliant with internationally acceptable standards.

Telecommunications is currently one of the most dynamic and rapidly evolving economic sectors worldwide, bringing about continuous technological and service innovations. Hence, it should not come as a surprise that the MNP introduction coincided in time with other important sectoral developments by mobile operators, such as the launch of $4 \mathrm{G}$ services, dramatic network coverage and service expansions, and widespread affordability of mobile communications, which all have had their unique as well as joint consequences on functioning of the market and behavior of an end user. One of the limitations of the present study is that it was hard to separate the effect of those developments from the impact of MNP, which would possibly require more complex econometric research. Nevertheless, the applied multistakeholder perspective approach had an advantage of analyzing the broader picture and still allowing to make sense out of that combined effect on the telecoms field, where MNP plays its concerted role.

Bearing in mind the above limitation, it is believed that the service availability alone has not had a considerable impact on individual market players and larger mobile communications segments in Belarus and Georgia. By itself, MNP has contributed to more active market competition and subscriber mobility, mostly benefitting the biggest service providers. However, changes over time in the performance of mobile operators, as detected by the analysis of scarce and inconsistent company-level data on operational and financial indicators, have occurred concurrently with the aforecited course of events. If taken separately, it would be assumed that MNP has had a scattered and limited indirect effect on the market and its participants.

On the consumer side, the conducted survey has revealed quite high rates of the mobile users' satisfaction with their existing service providers in both countries. However, there is yet willingness to change carriers if a different operator offers a more attractive deal in terms of better tariffs and quality of service. Besides, the respondents have disclosed strong attachment to their mobile phone numbers, which may create grounds for the increased MNP uptake in the future.

For the time being though, the usage rate within the surveyed country samples is low. Among a few of MNP users, many respondents stated that they are now better off with the new service provider compared to the previous one, even in the case if their average monthly bill for mobile services has 
increased after MNP in the range of $25-50 \%$. This finding indicates that consumers do not regard the size of their bills and underlying charges as a decisive factor for individual wellbeing and perceived utility associated with a particular mobile operator. It seems to be in contrast with a finding from the academic literature, whereby a large number of respondents to a consumer survey before the launch of MNP in Japan mentioned their high monthly bills as the most powerful motivation to switch mobile carriers (Otsuka and Mitomo, 2012). This kind of differences between countries rather reflects individual consumer preferences and is supportive of the study by Czajkowski and Sobolewski (2010), pointing out the importance of several non-price attributes while choosing a mobile carrier, such as the operator's brand name, the presence of close people's contacts on the same network, the overall size of an operator, etc.

From the same research by Otsuka and Mitomo (2012) on user benefits and operator costs of MNP in Japan, their suggested classification of direct and indirect benefits seems to fit also Belarusian and Georgian MNP users, with the following accrued benefits:

- Direct benefit of enjoying better services at a lower price;

- Indirect benefit of reduced call charges as a result of the competition increase.

Therefore, it can be concluded that the service availability has generally benefitted MNP users in Belarus and Georgia. The above indirect benefit might be accessible to non-MNP customers as well, as more intensive market competition is likely to drive prices down for everyone. However, half of the survey respondents (both with and without MNP experience) in each of the two countries found it difficult to answer the question on whether they believe that the mobile communications market in their country has overall gained from MNP in terms of more competitive service offerings, better tariffs and quality of service, etc.

\section{Key inferences and avenues for future research}

To sum up by referring to the central question of the given research, i.e.,

\section{Have MNP implementations had any substantial impact on the electronic communications markets of Belarus and Georgia?}

and by aggregating main findings of the multi-stakeholder analysis described herein, including views of the key parties concerned, it is concluded that MNP in both countries has not, on its own, significantly impacted the market and its players. The effects have rather been limited and indirect in nature through fostering market competition, enabling subscriber mobility without altering the phone number, and consequently benefitting MNP users with more favorable service and pricing conditions. All related consequences, such as reduction in mobile service prices and ARPU levels, change in the market structure, etc., have been the derivatives of a series of developments happening in parallel after the MNP launch.
This has been the first comprehensive, ex post research on the impact of MNP in Belarus and Georgia, at least among those that are publicly available. Besides, it has been the rare study in this domain, featuring a multi-stakeholder approach whereby the relevant data are collected from all involved parties, i.e., end users, mobile operators, and national regulatory/ policy-making authorities. The latter category is often omitted from the discussion, as the formerly carried out MNP research predominantly focuses on either mobile operator-level panel data or an end-user level survey data, or sometimes examines a combination of both. The given research has counted in views and considerations of that important stakeholder group in addition to mobile operator and consumer perspectives. Such an approach was believed to ensure a more inclusive feedback on the MNP effects and it seems to have met this expectation.

Despite the difference in research methods, the present study has delivered results that are generally consistent with those of the previous empirical attempts to capture the MNP effects in other countries. In line with the explanation by Telecommunications Management Group (2008), the current MNP frameworks in both Belarus and Georgia comply with all attributes of an ideal MNP regime, which "should be recipient network driven; use a centralized, neutrally administered database; feature direct routing; and have short lead times and low or no direct costs for the subscriber." The characteristics that form consumer choices of a mobile operator also seem to correspond to those identified by Czajkowski and Sobolewski (2010), the top three being (1) prices for on-net/ off-net calls charged by the operator, (2) presence of family member contacts on the same network, and (3) presence of friends and other close people contacts on the same network, with minor deviations in the order of importance between the two countries. The mobile company's brand name is not of that much significance among Belarusian and Georgian customers; however, when they feel happy with a particular service provider, they tend to become less sensitive towards prices charged for mobile calls.

As earlier research shows, there have been considerable variations in MNP patterns across countries. It is evident from highly heterogenous porting statistics from one country to another, and so the number of mobile portings cannot be regarded as a sole indicator for the success of MNP. Belarus and Georgia are not exceptions in this respect, with quite a low percentage of MNP users in the former country and the relatively large uptake of the service in the latter one. Hence, in order to assess the impact of MNP, certain other factors apart from mere porting statistics should be taken into account, which occurred at around the same timing with the MNP launch and had influenced the mobile telephony market. As such developments, the introduction of the $3.5 \mathrm{G}$ service and the entry of a new mobile operator were referred to by Otsuka and Mitomo (2012) in their research on the MNP implementation in Japan.

Aside from the above commonalities, there are explicit differences in findings between the given research and earlier studies on the effects of MNP, which are mainly attributable to the individual country and consumer specifics. According to Buehler at al. (2006), MNP pursues the following two 
objectives: (1) it removes barriers to switch service providers and thus directly benefits mobile customers, and (2) it provides equitable conditions for new players to enter the market and generate a sufficient customer base to be able to compete with incumbents. While there is evidence that the first objective has been achieved in the two studied countries, the second one has remained largely unmet, which is concluded from the latest market entrants' direct feedback during interviews and the subsequent analysis of their operational and financial performance indicators. The MNP availability in Belarus and Georgia has for the most part benefitted the first two biggest players by further solidifying their market positions. It is also in contrast with findings of several other studies, including by Cho at al. (2013), whereby MNP normally reduces market concentration by taking it away from the incumbent operators.

It is obvious that none of the applicable research studies is perfect and each has its distinct limitations, which may include the following, among others:

- A broad picture of the MNP effects is not provided;

- The change in price and consumer welfare as a result of MNP is not clearly articulated;

- The impact of the MNP policy provisions on market outcomes is not analyzed; and

- The reaction to MNP of different mobile operators in the same country is not captured.

The current multi-stakeholder approach applied herein helped to address three of the above four limitations, as follows:

- A broader and inclusive outlook on the impact of MNP was indeed analyzed through collecting and processing feedback from the major relevant parties all at once;

- The effect of the MNP policy objectives on the overall market outcomes was included by covering the voice of national regulatory and/ or policymaking authorities - an important stakeholder group that had often been excluded from the former research; and

- The attitudes toward MNP of all mobile operators in the country were considered, by thoroughly interviewing each of them and analyzing their operational and financial performance before and after the MNP launch.

The presented research also had its own limitations, the most important of which was the availability of consistent data, specifically on the mobile operator level. In any case, it still allowed reaching certain meaningful inferences. Nevertheless, there is always room for further research to build on top of the existing one. The following suggestions are possible directions in this regard:

1. To continue analyzing the available consumer dataset, as it represents a massive array of data on mobile users in Belarus and Georgia;
2. To make another effort to gather and analyze firmlevel data on mobile operators;

3. To conduct research on different subsets of mobile users, e.g., business and residential customers, prepaid and post-paid subscribers, etc., to reveal their specific preferences and experiences with respect to MNP;

4. To perform a similar study after several years from now, as the use of MNP, according to findings of the previous empirical research, tends to accelerate in the long run, supporting the argument that the length of time since the facility has been in place can be another feature to explore its impact on the effectiveness of MNP;

5. To carry out similar research in other post-Soviet countries that have implemented MNP after Belarus and Georgia, e.g., Moldova, Azerbaijan, Russia, Armenia and Kazakhstan, to compare the results and lessons learnt.

\section{Acknowledgment}

The author thanks Prof. Sam Dzever, Director of PhD in ICT Management program at Telecom Ecole de Management, and Dr. Bulat Sanditov, academic supervisor from the same school, for their continuous guidance during the research period (2013-2016). Special thanks are also extended to the author's personal and professional contacts, who enormously assisted in the data gathering efforts.

\section{References}

[1] R. Samarajiva, "Mobile Number Portability: Lessons from international experience," 2013, available at http://lirneasia.net/wpcontent/uploads/2013/07/Samarajiva_June2012.pdf.

[2] T. Iqbal, "Mobile Number Portability in South Asia," 2010, available at http://lirneasia.net/wp-content/uploads/2010/02/Mobile-2.0_MNP.pdf.

[3] W. Larkotey, D. Ansong, D. Damoah, and J. Abandoh-Sam, "Mobile Number Portability in Developing Countries: Its successes and failures. Case Study- West African Sub-Region," International Journal of Societal Applications of Computer Science, vol. 1, issue 1, 2012.

[4] T. Otsuka and H. Mitomo, "User benefits and operator costs of mobile number portability in Japan and impact on market competitiveness," Telecommunications Policy, 2012.

[5] M. Czajkowski and M. Sobolewski, "Measuring Network Effects in Mobile Telecommunications Markets with Stated-Preference Valuation Methods," 2010 [Conference Paper, 21 ${ }^{\text {st }}$ European Regional ITS Conference, Copenhagen 2010].

[6] Telecommunications Management Group, Inc. (TMG), "Mobile Number Portability Around the World," 2008.

[7] S. Buehler, R. Dewenter, and J. Haucap, "Mobile Number Portability in Europe," Telecommunications Policy, vol. 30, issue 7, pp. 385-399, 2006.

[8] D. Cho, P. Ferreira, and R. Telang, "The Impact of Mobile Number Portability on Price, Competition and Consumer Welfare," School of Information Systems and Management, Heinz College, Carnegie Mellon University, 2013. 
Armen Ghalumyan is an experienced ICT expert specializing in regulatory affairs and e-government, with project management experience in the mentioned fields. He was involved in a number of ICT-related assignments by the European Bank for Reconstruction and Development (EBRD) and the World Bank, addressing telecom sector developments in newly independent states. Since April 2015 he has been working as a home-based Senior Telecom and Postal Analyst for Cullen International, a Brussels-based independent regulatory intelligence provider covering telecoms, digital economy, media, postal regulation and competition law across Europe, Americas, the Middle East and North Africa. Mr. Ghalumyan holds Master's degrees in Information Technology Management from the Central European University Business School in Hungary, in Business Administration from the American University of Armenia, and in Radio Engineering from the State Engineering University of Armenia. At present he is a $\mathrm{PhD}$ in ICT Management candidate at Telecom Ecole de Management, a highly ranked public business school in France. His research covers the study of the impact of mobile number portability (MNP) implementations in a couple of post-Soviet countries. Mr. Ghalumyan is the sole author of three articles based on his research findings and a contributor to the EBRD's report on the Regional 2012 Electronic Communications Sector Comparative Assessment of 31 current and prospective countries of the Bank's operation. 